05

\title{
Термодинамические свойства жидкого цезия: поиск аномалий
}

\author{
(С) Л.Р. Фокин, Е.Ю. Кулямина \\ Объединенный институт высоких температур РАН, \\ 125412 Москва, Россия \\ e-mail: Ifokin@mail.ru, kulyamina.elena@gmail.com
}

Поступило в Редакцию 24 июня 2021 г.

В окончательной редакции 24 августа 2021 г.

Принято к публикации 1 сентября 2021 г.

\begin{abstract}
Объявленный в конце 90-х годов прошлого столетия полиморфизм жидкого цезия при атмосферном давлении в области температур $\sim 590 \mathrm{~K}$ в виде фазового перехода второго рода не находит подтверждения в новых экспериментальных работах и при компьютерном моделировании свойств. В то же время вопрос, носит ли изменение свойств жидкого цезия при уменьшении или увеличении плотности до двух раз монотонный характер или сопровождается различными аномалиями, нуждается в дальнейших исследованиях.
\end{abstract}

Ключевые слова: жидкий цезий, полиморфизм, фазовый переход второго рода, плотность, адиабатический термический коэффициент давления, структура.

DOI: 10.21883/JTF.2022.01.51854.192-21

\section{Введение}

В последние 20-30лет тема фазовых переходов в неупорядоченных средах, в жидкостях (liquid-liquid phase transition LLPT) является одним из важных направлений научных исследований. Обзор работ на эту тему опубликован, например, в статье 2018 г. Anisimov M.A. etal. „Thermodynamics of fluid polyamorphism“ [1]. Paнее критический анализ экспериментальных и теоретических исследований, посвященных проблеме фазовых переходов в жидкостях, был представлен в [2,3]. При этом для однокомпонентных систем рассматриваются в первую очередь фазовые переходы первого рода (ФП-1), когда две фазы сосуществуют. В настоящей работе мы рассматриваем другой вариант - фазовые переходы второго рода (ФП-2). Как отмечает И.И. Новиков, линия ФП-2 является для обеих фаз границей устойчивости, „образованной слившимися в одну линию спинодалями обеих фаз“" [4].

Классическим примером ФП-2 является жидкая сера, для которой такой переход наблюдает при атмосферном давлении и температуре $159^{\circ} \mathrm{C}$. Этому явлению посвящена обширная литература. По определению ФП-2, первые производные от термодинамического потенциала при $p=\mathrm{const}-$ плотность $\rho=1 / v$, энтропия $s-$ совпадают для обеих фаз, а скачком меняются вторые производные: коэффициент термического расширения (КТР) $\alpha=1 / v(d v / d T)_{p}$, коэффициент сжимаемости $\beta=-1 / v(d \nu / d p)_{T}$, теплоемкость $c_{p}=T(d s / d T)_{p}$. На линии ФП-2 выполняются уравнения Эренфеста, один из вариантов которых представлен ниже [5]:

$$
\begin{gathered}
d p / d T=-\left(\alpha^{\prime \prime}-\alpha^{\prime}\right) /\left(\beta^{\prime \prime}-\beta^{\prime}\right), \\
T(d p / d T)^{2}=-\left(c_{p}^{\prime \prime}-c_{p}^{\prime}\right) / v\left(\beta^{\prime \prime}-\beta^{\prime}\right) .
\end{gathered}
$$

Важно подчеркнуть, что для ФП-2 уравнения Эренфеста отражают связь скачков вторых производных так же, как для ФП-1 уравнение Клапейрона-Клаузиуса является связующим звеном для скачков плотности и энтропии сосуществующих фаз. Вблизи линии ФП-2 КТР, сжимаемость, теплоемкость с обеих сторон испытывают заметные изменения, которые, со своей стороны, служат предвестником фазового перехода. Для серы характерная картина изменения плотности и теплоемкости вблизи ФП-2 с двух сторон от $159^{\circ} \mathrm{C}$ (показана на рис. 1, заимствованном из работы [6]).

Для серы $p-T$ зависимость линии полимеризации от давления, где реализуется ФП-2, была исследована в работе [7]. На начальном участке значение производной $d T / d p=-0.18 \mathrm{~K} \cdot \mathrm{GPa}^{-1}$, что позволяет, используя зависимости (1), осуществить согласование опытных данных по теплоемкости и КТР сосуществующих фаз. Характерно, что в фундаментальном справочнике по термодинамическим свойствам веществ издания 1978 г. [8] для жидкой серы приводятся зависимости теплоемкости до

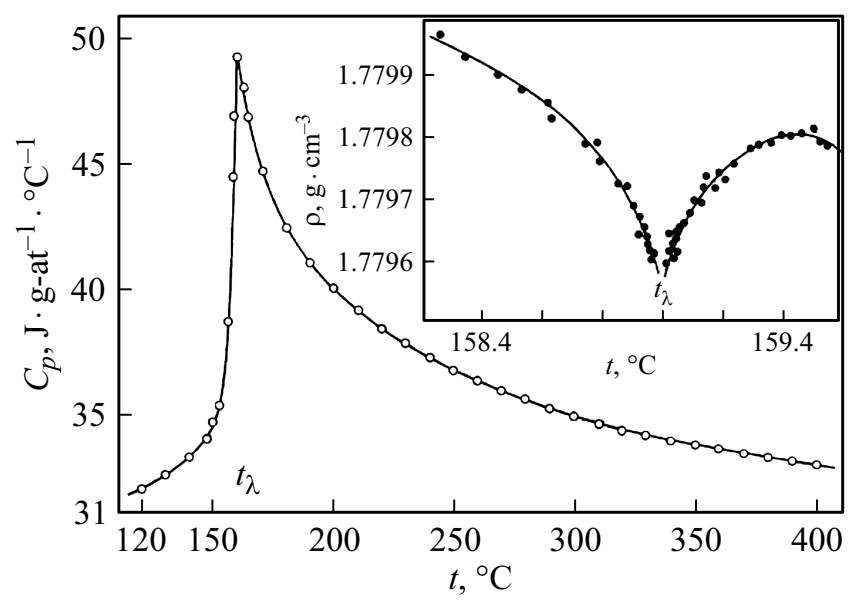

Рис. 1. Картина изменения плотности и теплоемкости жидкой серы с двух сторон относительно температуры ФП-2. 
и после $433 \mathrm{~K}$ (том 1, кн. 1, гл. 12), однако в кн. 2 в таблице свойств этот интервал температур отсутствует.

Во второй половине прошлого столетия были проведены обширные исследования теплофизических свойств жидкометаллических теплоносителей, в частности, щелочных металлов. При этом в ряде работ по термодинамическим свойствам жидкого цезия отмечаются некоторые особенности (аномалии) на температурной зависимости плотности насыщенной жидкости, которым авторы приписывают признаки ФП-2 [9-11]. В настоящей работе мы проводим анализ этих работ на фоне тех представлений и признаков о ФП-2 серы, которые были рассмотрены выше.

\section{1. Опытные данные о плотности и адиабатическом термическом коэффициенте давления жидкого цезия}

В 1965-70 гг. во вновь образованном Институте теплофизики Сибирского отделения АН в Новосибирске А.С. Басин и А.Н. Соловьев на установке, реализующей метод поглощения рентгеновских лучей, провели тщательные измерения плотности жидких свинца, галлия и цезия, для последнего 30 точек в интервале 307-1125 K [12]. В этой работе опытные данные о плотности жидкого галлия высокой чистоты в интервале температур 296-1408 К были „интерполированы отрезками трех прямых“. Отклонения опытных точек относительно сглаживающих зависимостей не превышали $\sim 0.1 \%$. Как отмечают авторы работы, в районе пересечения прямых при 505 и $1020 \mathrm{~K}$ были зафиксированы резкие изменения КТР, и „такие изломы должны быть видны на температурных зависимостях других свойств“. Здесь мы имеем дело с проблемой непрерывного или скачкообразного изменения термодинамических свойств и, в частности, с вопросом о том, в каком случае пересекающиеся прямые свойств на изолиниях могут заменить истинные непрерывные зависимости.

Позже для жидкого цезия А.С. Басин с помощью метода проникающего излучения провел подробные измерения плотности на изобарах 0.6-20 atm. при температурах до $1260^{\circ} \mathrm{C}$, около 220 опытных точек. Эти результаты измерений плотности были приведены к линии насыщения и аппроксимированы полиномом второй степени

$$
\begin{gathered}
\rho=1.747-0.056 x-6.2 \cdot 10^{-4} x^{2} \mathrm{~g} \cdot \mathrm{cm}^{-3}, \\
x=(t-200) / 100
\end{gathered}
$$

разбросом точек $\sim 0.2 \%$ относительно сглаживающей зависимости $[9,13]$.

При проведении экспериментов по плотности цезия методом проникающего излучения и анализе опытных данных А.С. Басин наблюдал заметное ( 15\%) падение КТР в интервале от температуры плавления до $150^{\circ} \mathrm{C}$

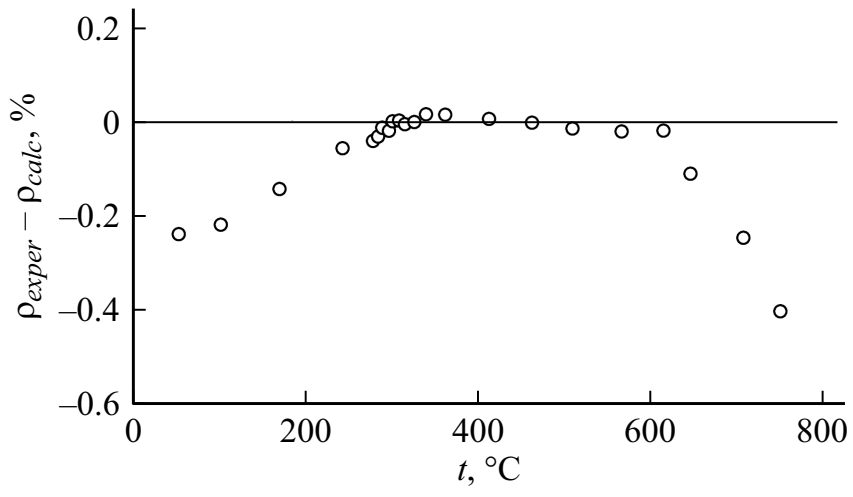

Рис. 2. Картина аномалий плотности цезия по данным работы [10].

и далее температурный рост КТР. Рассматривая это как аномалию свойств жидкости, автор в то же время не нашел достаточных аргументов, чтобы объявить пересечение линий плотности в этой области температур, как это сделано для галлия [12]. В справочных таблицах для плотности цезия в области $150^{\circ} \mathrm{C}$ [9] приведены данные, сглаженные „в пределах допустимых отклонений“, и зафиксирован минимум КТР на политерме плотности.

Практически через 10 лет, в 1979 г., признаки аномалий на бинодали жидкого цезия были заявлены в диссертации С.Н.Сковородько, подготовленной в ИВТАН под руководством Э.Э.Шпильрайна и В.А. Фомина [10]. В работе для измерения плотности жидкого цезия и ряда сплавов щелочных металлов был использован метод пикнометра переменной массы. Ампула с жидким металлом находилась в тщательно термостатированном объеме печи и имела выход в область комнатных температур через капилляр. Металл в ампуле и металлоприемник находились под давлением инертного газа. При нагреве ампулы избыток металла через капилляр сливался в металлоприемник, который в нужный момент перекрывался пробкой, а вытекший металл после разгерметизации установки вымывался. Взвешивание ампулы с металлом до и после опыта и учет необходимых поправок позволяли определять плотность металла при температуре печи. В этом весьма трудоемком эксперименте для плотности цезия на линии насыщения в интервале температур $322-1074$ К были получены 24 опытные точки. Погрешность данных, по оценкам авторов, не превышала $0.15 \%$. В диссертации аномалии цезия на политерме плотности показаны в виде трех отрезков опытных данных $\rho(T)_{\text {exper }}$, пересекающих линейную зависимость $\rho(T)_{\text {calc }}$ плотности цезия из работы [14] (рис. 2).

На рисунке при температуре $\sim 600 \mathrm{~K}$ мы видим резкое изменение наклона линии первых 12 точек и затем через 8 точек в районе $900 \mathrm{~K}$ новое изменение наклона в другую сторону. Надо отметить два обстоятельства. Первое - рассматриваемые особенности поведения опытных данных лежат в границах $0.2 \%$, что практически соответствует авторской оценке их 


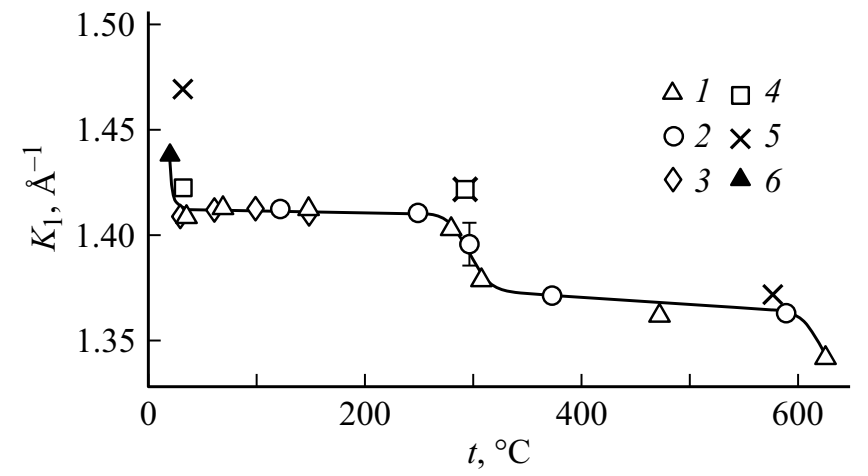

Рис. 3. Положение координаты $K_{1}\left(S_{\max }\right)$ первого максимума структурного фактора в зависимости от температуры по экспериментальным данным о рассеянии нейтронов в жидком цезии [16]. Обозначения: 1 - первая экспериментальная серия, 2 - вторая, 3-6 - данные других авторов.

погрешности. Второе - на рисунке в пределах $0.2 \%$ не виден разброс опытных данных относительно линейных зависимостей, что кажется странным для такого сложного эксперимента. К сожалению, мы не знаем, какие и в каких пределах менялись условия эксперимента, и поэтому не имеем доказательств воспроизводимости и достоверности рассматриваемых данных. Для жидкого цезия в диссертации первый скачок производных $d \rho / d T$ в районе $600 \mathrm{~K}$ рассматривается как фазовый переход второго рода ФП-2. Опытные данные по плотности из работы [10] позже были приведены в [15], где они представлены полиномом второй степени

$$
\rho=1.747-0.056 x-6.2 \cdot 10^{-4} x^{2} \mathrm{~g} \cdot \mathrm{cm}^{-3},
$$

где $x=T / 1000$.

Отклонения опытных точек относительно этой зависимости лежат в пределе $0.1 \%$ и носят волнообразный характер.

Естественно предполагать, что особенности в поведении теплофизических свойств на изолиниях вещества сопровождаются в той или иной степени изменениями в его структуре. В Институте атомной энергии им. И.В. Курчатова на нейтронном дифрактометре были проведены исследования структуры жидкого цезия в интервале температур $35-650^{\circ} \mathrm{C}$ [16]. Металл высокой чистоты находился в тонкостенной ванадиевой ампуле. Диапазон волновых чисел $K=4 \pi \sin (\theta / 2) / \lambda$, где $\lambda=1.37 \AA-$ длина волны излучения, $\theta-$ угол рассеяния, лежит в интервале $0.13-6.3 \AA^{-1}$. Опытные данные для угловой зависимости интенсивности рассеяния на изотермах позволили восстановить значения структурного фактора $S(K)$ и зафиксировать положения первого максимума $K_{1}\left(S_{\max }\right)$ на шкале волновых чисел. В районе $230^{\circ} \mathrm{C}$ авторы наблюдали резкое изменение (падение) значений $K_{1}(t)$ с ростом температуры (рис. 3), что, по их мнению, являлось подтверждением аномалии плотности жидкого цезия в этой области температур.
С другой стороны, на рис. 3 мы видим лишь резкое изменение положения первого максимума $K_{1}\left(S_{\max }\right)$, и, надо отметить, не более того.

Одновременно нейтронографические исследования изменений структуры в жидком рубидии в зависимости от температуры были проведены в Институте физики АН Латвийской ССР $[17,18]$. Методика, интервал волновых чисел, интервал температур подобны тем, что и в работе [16]. Для рубидия в районе $300^{\circ} \mathrm{C}$, как и для цезия в области $230^{\circ} \mathrm{C}$, авторы наблюдали резкое изменение положения первого максимума структурного фактора $K_{1}\left(S_{\max }\right)$. В статье [17] на рисунке показаны новые опытные данные о плотности жидкого рубидия [10] в интервале температур $100-750^{\circ} \mathrm{C}$. Эти данные аппроксимированы тремя пересекающимися линиями, и первая точка пересечения $\sim 300 \mathrm{~K}$. В рассматриваемых работах наблюдаемые особенности поведения структуры и плотности на политермах жидких рубидия и цезия качественно носят общий характер.

Надо отметить, что нейтроно- и рентгенографические исследования структуры жидких щелочных металлов, в частности цезия, в широком интервале температур и давлений проводились и проводятся специалистами в разных странах (см., например, $[19,20])$. В этих работах, как правило, основное внимание уделяется анализу свойств жидких металлов в околокритической области их состояния. В то же время, например, в работе [20] на основе данных о рассеянии мощного синхротронного $\gamma$-излучения на жидком цезии построены изотермы структурного фактора $S(K)$ и радиальной функции распределений $g(R)$ в широком интервале температур 313-1873 K. Можно видеть (рис. 4), например, что положение первого максимума $R\left(g_{\max }\right)$ в интервале температур 313-773 К при уменьшении плотности цезия от 1.82 до $1.60 \mathrm{~g} \cdot \mathrm{cm}^{-3}$ изменяется монотонно и лишь незначительно уменьшается аналогично $K_{1}\left(S_{\max }\right)$. Эти наблюдения не подтверждают аномалии в поведении плотности и структуры жидких цезия и рубидия, которые рассматривались выше в работах $[16,17]$.

В статистической теории жидкостей связь структурных характеристик и теплофизических свойств выражается через интегральные зависимости. При этом многие проблемы такого анализа связаны с трудностями определения структуры при малых значениях волновых

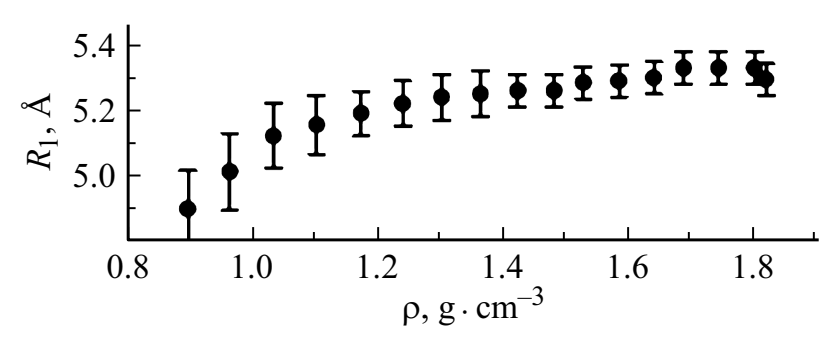

Рис. 4. Положение первого максимума $R_{1}$ радиальной функции распределения в зависимости от плотности жидкого цезия по данным работы [20]. 
чисел $K$. В этом отношении представляет особый интерес работа [21], в которой для многих, в частности для щелочных металлов, приведены значения структурного фактора $S(K)$ при малых значениях чисел $K>0.05 \AA^{-1}$ в области температур на $200-300^{\circ} \mathrm{C}$ выше температуры плавления.

Объявленные в работах $[9,10,16-18]$ положения о возможности реализации ФП-2 в жидком цезии на линии насыщения вызвали интерес у многих исследователей. В частности, на этой почве возникло многолетнее сотрудничество группы исследователей свойств жидких металлов из Объединенного института высоких температур РАН (ИВТАН) и сотрудников кафедры молекулярной физики физического факультета МГУ. К тому времени на кафедре под руководством проф. Л.П. Филиппова проводились активные исследования теплофизических свойств твердых и жидких металлов с использованием методов периодического нагрева проводников [22], и имелся опыт исследований теплоемкости жидкого цезия в широкой области температур [23]. Кроме того, на кафедре проводились измерения термодинамических свойств диэлектрических жидкостей (воды, углеводородов) с использованием упруготермического эффекта, основанного на соотношении Томсона [24] для описания процесса изоэнтропического сжатия среды

$$
\gamma=(\partial T / \partial p)_{S}=T \alpha_{p} /\left(\rho c_{p}\right),
$$

где $\gamma$ - адиабатический термический коэффициент давления (АТКД), $s$ - энтропия, $\alpha_{p}-$ изобарный коэффициент термического расширения, $c_{p}$ - удельная изобарная теплоемкость. На кафедре была создана установка, которая по замыслам авторов должна была подтвердить или опровергнуть наличие ФП-2 в жидком цезии в области температур $\sim 590 \mathrm{~K}$ на основе идеи периодического адиабатического упруготермического воздействия на вещество. В то же время, анализируя структуру соотношения Томсона, можно видеть, что именно в области ФП-2 особенности (аномалии) поведения КТР и теплоемкости (рис. 1 для серы) могут скомпенсировать друг друга, но здесь важна практика работы на конкретном объекте.

В университете на кафедре для исследования упруготермического эффекта в жидком цезии была создана экспериментальная установка, основные характеристики которой приведены в работе [25]. Реализована схема сравнительных измерений температурного отклика при одновременном квазиадиабатическом сжатии металла и эталонной жидкости (n-гексан). Вещество находится в металлическом стакане, в который снизу введен капилляр диаметром $3 \mathrm{~mm}$, к крышке которого изнутри приварена хромель-алюмелевая (ХА) термопара диаметром $0.2 \mathrm{~mm}$. Импульсное периодически изменяемое с частотой $\sim 3 \mathrm{~Hz}$ давление $\sim 1 \mathrm{MPa} \mathrm{жидкости} \mathrm{создает-}$ ся гидравлическим генератором, производящим рост и сброс до нуля. Изменяемое давление жидкости через сильфон передается рабочему веществу. Оценки по
Отклонения $\Delta=\rho_{\text {exper }}-\rho_{\text {calc }}$ опытных данных $\rho_{\text {exper }}$ от аппроксимирующего полинома второй степени $\rho_{\text {calc }}$ при температурах 578-608 K на этапе охлаждения

\begin{tabular}{c|r|r|r|r|r|r|r}
\hline$T, \mathrm{~K}$ & 578.5 & 583.2 & 588.0 & 593.0 & 598.0 & 603.2 & 608.5 \\
\hline$\rho_{\text {exper }}, \mathrm{kg} \cdot \mathrm{m}^{-3}$ & 1689.8 & 1682.7 & 1677.6 & 1679.7 & 1674.3 & 1673.8 & 1667.5 \\
\hline$\Delta, \mathrm{kg} \cdot \mathrm{m}^{-3}$ & 4.0 & -0.3 & -2.9 & 2.0 & -0.7 & 1.7 & -1.6
\end{tabular}

уравнению Томсона (4) показывают, что при таком импульсе давления в жидком цезии в интервале 400-700 K возникает температурный отклик $0.2-0.7 \mathrm{~K}$ и на ХА термопаре напряжение $U=10-20 \mu \mathrm{V}$, которое легко регистрируется современной высокочувствительной аппаратурой. По существу, в этом варианте проведение АТКД эксперимента сводится к измерению отношения $U_{\mathrm{Cs}} / U_{\text {Stand }}$ цезия и эталонной жидкости и учету необходимых поправок. В процессе работы установки была усовершенствована схема крепления термопар, разработана процедура учета неадиабатичности и т.п. [23]. Определенный этап этих исследований отражен в публикациях [26,27]. В частности, в статье [26] для интервала температур 403-605 К приведена таблица опытных данных $U_{\mathrm{Cs}} / U_{\text {Stand }}$, значения поправок на неадиабатичность и коэффициенты $\gamma=(\partial T / \partial p)_{S}$. Как ни странно, в этой и подобных публикациях авторов отсутствует информация о качестве металла до и после проведения эксперимента, схема заполнения установки, анализ на наличие газовых примесей в цезии. В работе на основе табличных данных построены графики $U_{\mathrm{Cs}}(T) / U_{\mathrm{Stand}}\left(T_{0}\right)$ для двух серий, на которых виден скачок значений в районе $590 \mathrm{~K}$ и изменение монотонного хода функции (рис. 5).

Почему в статье не приводятся более подробные данные в этой области температур, не ясно. Тем не менее после обсуждения этих АТКД экспериментальных данных авторы отмечают, что „наблюдаемые аномалии теплофизических свойств цезия несут на себе черты фазового перехода 2-го рода“. В другой статье [27] авторы

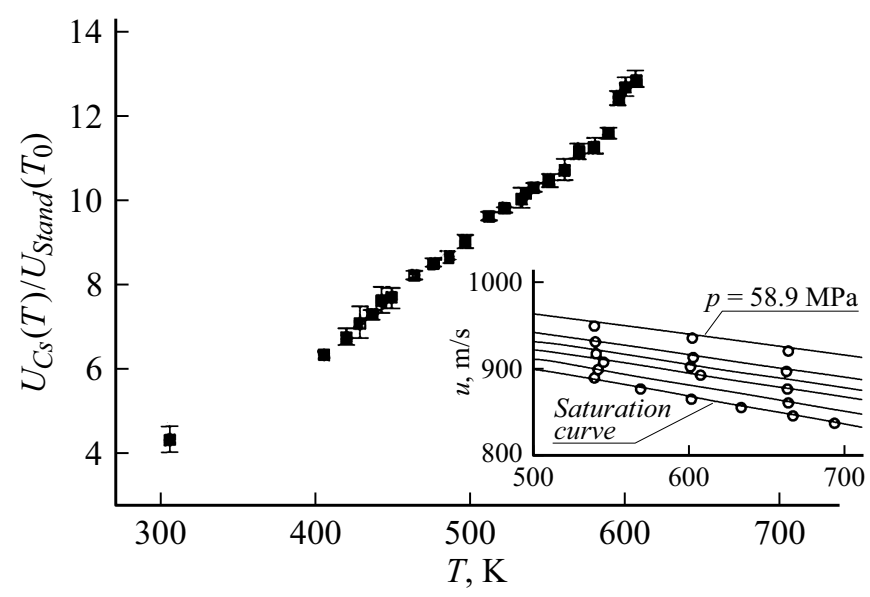

Рис. 5. Опытные данные по АТКД [26] и скорости звука в жидком цезии [28]. 
еще раз рассматривают все имеющиеся у них аргументы: особенности температурной зависимости плотности [10] и вязкости жидкого цезия, опытные данные об изменении структуры [16-18] и, главное, рассмотренные выше результаты измерений АТКД (рис. 5), и в заключение еще раз декларируют признаки ФП-2 в области $590 \mathrm{~K}$.

На вставке рис. 5 показан фрагмент опытных данных о скорости звука в жидком цезии на линии насыщения $u(T)$ и на изобарах до $600 \mathrm{bar}$ [28]. На температурных зависимостях этих данных трудно усмотреть какие-либо особенности, которые бы подтверждали нерегулярный характер поведения АТКД в области $590 \mathrm{~K}$ в работах $[26,27]$ и тем самым предполагаемый ФП-2.

\section{2. Расчетные работы с признаками аномалий в жидком цезии}

Гхати и Бахадори (Университет Шираз, Иран) проводили анализ свойств жидкого цезия на линии насыщения и при давлениях до 600 bar, используя метод линеаризованных изотерм

$$
\left[(Z-1) V^{2}\right]_{T}=C+B V
$$

где $Z=p V /(R T)$ - фактор сжимаемости, $p$ - давление, $R$ - газовая постоянная, $B$ и $C-$ коэффициенты, связанные с параметрами потенциальной функции Леннарда-Джонса $m-n$, глубиной потенциальной ямы $\varepsilon$ и диаметром столкновений $\sigma[29,30]$. Используя таблицы опытных данных о плотности жидкого цезия из работы [28] в интервале температур 400-1900 K с шагом $100 \mathrm{~K}$, авторы оценили значения коэффициентов $B(T)$ и $C(T)$ на изотермах и построили графики зависимостей $\varepsilon(T)$ и $\sigma(T)$. На графике $\varepsilon(T)$ имеются две области немонотонного поведения точек. Первая в районе $1350 \mathrm{~K}$, которую авторы относят к началу перехода металл-неметалл в жидком цезии, и вторая, слабо выраженная, - в районе $600 \mathrm{~K}$, которую относят к области возможного ФП-2, обсуждаемого в работах $[16,17,26,27]$. Естественно, этот результат, полученный сложным расчетным путем, заслуживает внимания. Однако при шаге изотерм $100 \mathrm{~K}$ в работе [28] нужна большая осмотрительность, чтобы за этой немонотонностью функции $\varepsilon(T)$ видеть фазовый переход.

Работы по моделированию структуры и свойств жидких металлов и сплавов (топологически неупорядоченные системы) проводятся в Екатеринбурге - ИМЕТ УрО РАН, в Челябинске - ЮрГУ, в Ижевске - Физтех УрО РАН. Заметное место в этих работах занимают жидкие щелочные металлы как тестовые объекты с более простой по отношению к переходным металлам электронной системой, для которых имеются опытные данные о свойствах, вплоть до критических температур, при значительном изменении плотности на линии насыщения. Характерной чертой проводимых расчетов является широкое использование методов топологического анализа структур с помощью многогранников Вороного

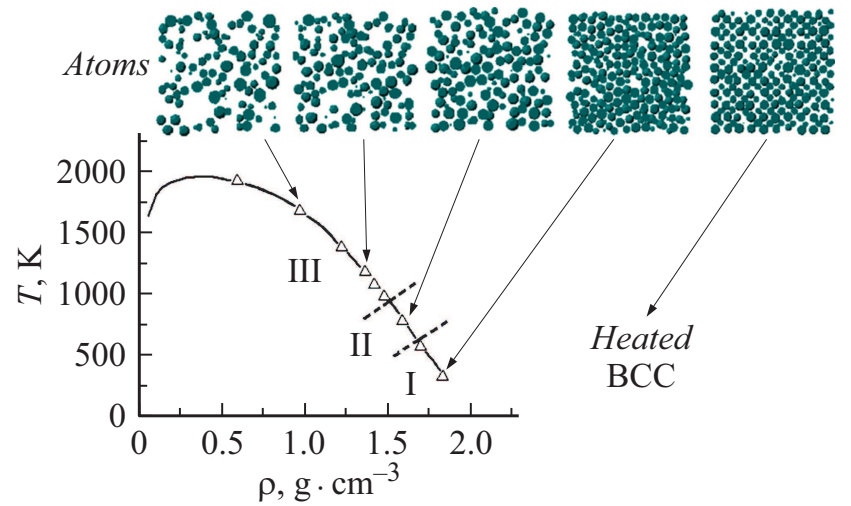

Рис. 6. Структурные фрагменты жидкого цезия и условные границы зон [32].

и симплексов Делоне. Многие вопросы методики и детали этих расчетов приводятся, например, в монографии [31]. Характерная картина изменения структуры жидкого цезия на линии насыщения, полученная в результате моделирования, показана на рис. 6 [32].

На рисунке выделены три зоны: плотная жидкость I, переходная область II и рыхлая жидкость III. Между зонами проведены четкие границы: I-II $590 \mathrm{~K}, 1.6 \mathrm{~g} \cdot \mathrm{cm}^{-3}$, II-III $930 \mathrm{~K}, 1.46 \mathrm{~g} \cdot \mathrm{cm}^{-3}$. Первая граница отождествляется с температурой ФП-2 (рис. 5), а сам фазовый переход рассматривается как установленный факт [26,27].

Д.К. Белащенко опубликовал серию статей, посвященных моделированию свойств жидких металлов в широком интервале температур и давлений. В качестве исходных для восстановления потенциалов были использованы опытные данные по структуре, стационарному сжатию и ударным волнам. В последнее время для потенциала взаимодействия автором последовательно применяется модель погруженного атома, когда к традиционному парному потенциалу добавляется влияние окружающей среды [33]. Что касается жидкого цезия, Д.К. Белащенко опубликовал статью „О структурном превращении в жидком цезии“, в которой рассматривается проблема полиморфизма в жидком цезии при давлениях $\sim 4 \mathrm{GPa}$ и при температуре $220^{\circ} \mathrm{C}$ [34]. Эта статья служит реакцией на публикацию 2005 г., в которой были представлены опытные данные о рассеянии рентгеновских лучей в цезии при указанных выше параметрах [35]. Анализируя изменение структурного фактора и радиальной функции распределения на изотерме в зависимости от давления, авторы [35] в районе $4 \mathrm{GPa}$ зафиксировали резкое уменьшение объема (до $17 \%$ ) и переход типа ФП1. Надо отметить, что в этой работе все данные о плотности носят расчетный характер. Такой результат для относительно простой системы на фоне других работ по полиморфизму жидких неупорядоченных систем имел принципиальное значение и косвенно подтверждал возможность возникновения ФП-2 в цезии при атмосферном давлении, которую мы обсуждали выше. Анализируя [35], Д.К. Белащенко, опираясь на свой многолетний 
опыт работы с такими экспериментальными данными и проведенные им расчеты, отметил, что некорректность результатов этих дифракционных исследований может быть связана с неверными определениями объема и координационных чисел в жидком цезии. Основной вывод проведенного анализа - „никаких признаков структурных превращений не обнаружено“. Прошло четыре года. В новой экспериментальной работе, но теперь не по рассеянию, а по поглощению рентгеновских лучей, в рассматриваемой области параметров признаки ФП-1 для жидкого цезия не были обнаружены [36].

Структурные и фазовые превращения в неупорядоченных системах являются одним из направлений работ Института физики высоких давлений РАН (ИФВД). Ю.Д. Фомин и соавторы из ИФВД в 2018 г. опубликовали результаты компьютерного моделирования методами молекулярной динамики (МД) термодинамических свойств жидкого цезия при невысоких давлениях для тестирования предполагаемого полиморфизма в районе $590 \mathrm{~K}$ [37]. МД моделирование проводилось в NpT-ансамбле (isothermal- isobaric ensemble) на массиве 3456 частиц. В качестве потенциала использован потенциал погруженного атома из работы [34]. Рассчитаны массивы pVT-данных и значения энтальпии на изобарах в интервале $300-1200 \mathrm{~K}$, при их дифференцировании значения КТР, теплоемкости $C_{P}$ и АТКД (4). Рассчитанные коэффициенты до $900 \mathrm{~K}$ качественно согласуются с опытными данными. На графиках в области $600 \mathrm{~K}$ можно видеть небольшие нарушения монотонности поведения КТР и теплоемкости, которые автоматически отражаются и на поведении АТКД. По мнению авторов, проведенные ими расчеты не подтверждают заявленный полиморфный переход жидкость-жидкость в работах $[26,27]$ с оговоркой, что причины появления „шумов“ на политермах свойств в области $600 \mathrm{~K}$ нуждаются в дальнейших исследованиях, с чем трудно не согласиться.

\section{Заключение}

Прошло 20 лет со времени появления основных публикаций [26,27], в которых рассматривалась аномалия свойств жидкого цезия при атмосферном давлении „по типу фазового перехода второго рода““. С тех пор новые опытные данные, подтверждающие выдвинутую гипотезу, не были опубликованы.

Исследования плотности жидких металлов и сплавов в широком интервале температур методом поглощения рентгеновских лучей, начатые в свое время в Институте теплофизики СО РАН А.С. Басиным $[9,12,13]$, активно проводятся в наше время. В 2020 г. сотрудниками института опубликована работа, посвященная исследованию плотности жидкого сплава $\mathrm{Cs}-\mathrm{Bi}$ при температурах до $1000 \mathrm{~K}$, в которой отдельно приводятся данные для жидкого цезия [38]. Металл высокой чистоты находился в стальном тонкостенном контейнере. Температура измерялась термопарой ХА. Публикации авторов со- держат детали экспериментов и оценки погрешностей опытных данных. Мы имели возможность более детально проанализировать опытные данные для цезия при нагреве и охлаждении в интервале 500-700 К. Результаты измерений плотности прямого и обратного хода совпадают в пределах $0.2-0.3 \%$. Принципиально важно то, что отклонения опытных данных от сглаживающей зависимости на каждом этапе носят случайный характер, и выделить участок систематических отклонений, который бы демонстрировал некоторую аномалию в поведении плотности, нет оснований (см. таблицу).

Анализируя эти данные, мы не видим оснований в рассматриваемом интервале температур для выделения двух пересекающихся участков политермы плотности, как это сделано в работе [10] (рис. 2), и тем самым подтверждения реализации ФП-2.

В это же время на физическом факультете МГУ были проведены новые исследования упруготермического эффекта в жидком цезии по несколько уточненной по сравнению с [26,27] методике в интервале температур 320-620 K [39]. Опыты проводились короткими сериями. При анализе результатов авторы не зафиксировали ожидаемый скачок АТКД в области $590 \mathrm{~K}$, подчеркивая одновременно повышенный разброс опытных данных в интервале $550-600 \mathrm{~K}$.

Тем самым новые опытные данные о структуре [20] и плотности [38], а также результаты компьютерного моделирования [37] свойств жидкого цезия при атмосферном давлении в области $590 \mathrm{~K}$ не подтверждают предполагаемый полиморфизм в форме ФП-2, который был заявлен в работах $[10,26,27]$.

По-видимому, реализация ФП-2 на термодинамической поверхности жидких металлов маловероятна. В то же время появление различных аномалий в виде экстремумов на изолиниях свойств вполне возможно. Например, для жидкого цезия в эксперименте зафиксирован максимум скорости звука $u_{\max }$ на изотерме $500 \mathrm{~K}$ при давлении $\sim 2 \mathrm{GPa}[40]$, и задача заключается в том, чтобы построить $(p, T)_{u}$-линию в широком интервале параметров, как это сделано для максимумов плотности воды в нормальном и метастабильном состояниях [41].

Надо отметить, что при проведении исследований свойств жидкого цезия в широком интервале параметров все авторы явно или неявно имеют в виду работы по сжимаемости и фазовой диаграмме твердой фазы. Известно, что при комнатной температуре при сжатии цезий испытывает переходы - вначале структурный ОЦК-ГЦК в районе $21 \mathrm{kbar}$, и затем электронный $5 s-5 d$ при давлении $\sim 42 \mathrm{kbar}[42,43]$. И все же, рассматривая структурные и электронные свойства на бинодали жидкой фазы цезия, мы находимся в другой области плотностей фактора упаковки атомов и структуры электронной подсистемы.

Проблема полиморфизма веществ в жидком состоянии сохраняет актуальность, что нашло отражение, например, в новом обстоятельном обзоре на эту тему [44], где представлены итоги экспериментальных и 
теоретических исследований фазовых переходов в однокомпонентных жидких и аморфных средах, в основном на уровне 2019 г. В статье [44] проанализированы новые уникальные опытные данные по фазовой $T-p$-диаграмме серы [45]. Диаграмма включает линию ФП-2 и ее пересечение с линией равновесия жидкой и твердой фаз. Выше по давлению экспериментально зафиксирована линия ФП-1 двух полимерных жидкостей, которая заканчивается критической точкой с параметрами $1100-1200 \mathrm{~K}$ и 2.1-2.2 GРа. Это вторая, после переохлажденной воды, критическая точка в жидкой фазе.

\section{Благодарности}

Мы благодарны Р.А. Хайрулину (ИТ СО РАН), который познакомил нас с новыми опытными данными по плотности жидкого цезия [38].

\section{Конфликт интересов}

Авторы заявляют, что у них нет конфликта интересов.

\section{Список литературы}

[1] M.A. Anisimov, M. Duska, F. Cauplin, L.E. Amrhein, A. Rosenbaum, R.J. Sadus. Phys. Rev. X, 8(1), 011004 (2018). DOI: 10.1103/PhysRevX.8.011004

[2] М.Г. Васин, В.И. Ладьянов. Вестник Удмуртского ун-та. Сер. физика. (4), 99 (2005).

[3] М.Г. Васин, В.А. Стекольщиков, В.И. Ладьянов. Вестник Удмуртского ун-та. Сер. физика. (4), 93 (2006).

[4] И.И. Новиков. ТВТ, 41 (3), 366 (2003). [I.I. Novikov. High Temperature, 41 (3), 312 (2003).

DOI: 010.1023/A:1024278207716]

[5] И.И. Базаров. Термодинамика (ГИФМЛ, М., 1961)

[6] А.Г. Кобзуненко, К.И. Кугель, Л.А. Макарова, М.А. Середницкая, Н.А. Татиашвили. ТВТ, 29 (5), 903 (1991). [A.G. Kobzunenko, K.I. Kugel', L.A. Makarova, N.A. Tatiashvili. High Temperature, 29 (5), 712 (1991).]

[7] K. Brollos, G.M. Schneider Ber. Bunsenges. Phys. Chem., 78|,(3), 296 (1974). DOI: 10.1002/bbpc.19740780315

[8] В.П. Глушко, Л.В. Гурвич, Г.А. Бергман, И.В. Вейц, В.А. Медведев. Термодинамические свойства индивидуальных веществ (Наука, М., 1978), т. 1, кн. 1,2.

[9] А.С. Басин. Автореф. канд. дисс. (Институт теплофизики СО АН СССР, Новосибирск, 1970)

[10] С.Н. Сковородько. Канд. дисс. (Институт высоких температур АН СССР, М., 1979)

[11] V.A. Alekseev, L.A. Blagonravov, F. Modhen, I.V. Samorodskaja. J. Non-Cryst. Solids, 156-158, (2), 659 (1993). DOI: 10.1016/0022-3093(93)90041-U

[12] А.С. Басин, А.Н. Соловьев ПМТФ, 6, 83 (1967).

[13] А.С. Басин. В сб.: Исследование теплофизических свойств веществ, под ред. С.С. Кутателадзе (Наука, Новосибирск, 1970)

[14] Э.Э. Шпильрайн, К.А. Якимович. ТВТ, 5 (2), 239 (1967).

[15] Э.Э. Шпильрайн, В.А. Фомин, С.Н. Сковородько, А.Г. Мозговой. ЖФХ, 75 (12), 2288 (2001).
[16] Ю.И. Шарыкин, В.П. Глазков, С.Н. Сковородько, В.А. Соменков, В.А. Фомин, С.Ш. Шильштейн, Э.Э. Шпильрайн. ДАН СССР, 244 (1), 78 (1979).

[17] А.Ю. Астапкович, Е.М. Иолин, Е.Н. Козлов, В.О. Николаев, С.Н. Сковородько, В.А. Фомин, С.Е. Циркунова, Ю.И. Шарыкин, Э.Э. Шпильрайн. ДАН СССР, 263 (1), 73 (1982).

[18] В.О. Николаев. Автореф. канд. дисс. (Институт высоких температур АН СССР, М., 1986)

[19] R. Winter, T. Bodensteiner. High Press. Res., 1 (1), 23 (1988). DOI: $10.1080 / 08957958808202478$

[20] K. Matsuda, S. Naruse, K. Hayashi, K. Tamura, M. Inui, Y. Kajiharal. J. Phys.: Conf. Ser., 98 (1), 012003 (2008). DOI: $10.1088 / 1742-6596 / 98 / 1 / 012003$

[21] Y. Waseda. Z. Naturforsch, 38a, 509 (1983).

[22] Л.П. Филиппов. Измерения теплофизических свойств веществ методом периодчческого нагрева (Энергоатомиздат, М., 1984)

[23] Л.А. Благонравов, Л.П. Филиппов, В.А. Алексеев, B.Н. Шнерко. ИФЖ, 44 (3), 438 (1983). [L.A. Blagonravov, L.P. Filippov, V.A. Alekseev, V.N. Shnerko. J. Eng. Phys. Thermophys., 44 (3), 304 (1983). DOI: 10.1007/BF00827368]

[24] Л.П. Филиппов, В.А. Стасенко, Л.А. Благонравов. Измерительная техника, 1, 48 (1984).

[25] Л.А. Благонравов, Модхен Фейссал. ПТЭ, 4, 167 (1991).

[26] Л.А. Благонравов, Л.А. Орлов, С.Н. Сковородько, В.A. Алексеев. TBT, 38 (4), 566 (2000). [L.A. Blagonravov, L.A. Orlov, S.N. Skovorod'ko, V.A. Alekseev. High Temperature, 38 (4), 542 (2000). DOI: 10.1007/BF02755799]

[27] L.A. Blagonravov, S.N. Skovorod'ko, A.S. Krylov, L.A. Orlov, V.A. Alekseev, E.E. Shpilrain. J. Non-Cryst. Solid., 277 (2-3), 182 (2000). DOI: 10.1016/S0022-3093(00)00315-X

[28] V.F. Kozhevnikov, S.P. Naurzakov, A.P. Senchenkov. J. Moscow Phys. Soc., 1, 171 (1991).

[29] M.H. Ghatee, M. Bahadori. J. Chem. Phys. B, 105 (45), 11256 (2001). DOI: 10.1021/jp011592q

[30] M.H. Ghatee, M. Bahadori. Fluid Phase Equilib., 233 (2), 151 (2005). DOI: 10.1016/j.fluid.2005.04.001

[31] Б.Р. Гельчинский, А.А. Мирзоев, А.Г. Воронцов. Вычислительные методы микроскопической теории металлических расплавов и нанокластеров (Физматлит, М., 2011)

[32] A. Vorontsov, D. Kutz. EPJ Web of Conferences, 15, 01004 (2011). DOI: 10.1051/epjconf/20111501004

[33] Д.К. Белащенко. УФН, $\mathbf{1 8 3}(12), 1286$ (2013). [D.K. Belashchenko. Physics-Uspekhi, $\mathbf{1 8 3}(12), 1176$ (2013). DOI: 10.3367/UFNe.0183.201312b.1281]

[34] Д.К. Белащенко. ЖФХ, $88(9), \quad 1373$ (2014). [D.K. Belashchenko. Rus. J. Phys. Chem. A, $88(9)$, 1533 (2014). DOI: 10.1134/S0036024414090052]

[35] S. Falconi, L. Lundegaard, C. Hejny, M. MacMahon. Phys. Rev. Lett., 94 (12), 125507 (2005). DOI: 10.1103/PhysRevLett.94.125507

[36] T. Hattori. Phys. Rev. B, 97 (10), 100101(R) (2018). DOI: 10.1103/PhysRevB.97.100101

[37] Yu.D. Fomin, E.N. Tsiok, V.N. Ryzhov. Phys. Chem. Liq., 57 (5), 650 (2019). DOI: 10.1080/00319104.2018.1509969

[38] R.A. Khairulin, R.N. Abdullaev, S.V. Stankus. Phys. Chem. Liq., 58 (2), 143 (2020). DOI: $10.1080 / 00319104.2018 .1553042$

[39] L.A. Blagonravov, A.A. Modenov. J. Phys.: Conf. Ser., 1385, 012043 (2019). DOI: 10.1088/1742-6596/1385/1/012043 
[40] F. Decremps, S. Ayrinhac, M. Gauthier, D. Antonangeli, M. Morand, Y. Garino, P. Parisiades. Phys. Rev. B, 98 (18), 184103 (2018). DOI: 10.1103/PhysRevB.98.184103

[41] Л.Р. Фокин. ЖФХ, $92(10), \quad 1450 \quad$ (2018). DOI: $10.1134 / \mathrm{S} 004445371810007$ [L.R. Fokin. Rus. J. Phys. Chem. A, 92 (10), 1877(2018).

DOI: $10.1134 / \mathrm{S} 0036024418100072]$

[42] M. Trömel, S. Hübner, K. Taxel. Z. Naturforsch. B, 58b, 1147 (2003).

[43] E.Yu. Tonkov, E.G. Ponytovsky. Phase Transition of Elements Under High Pressure (CRC Press, 2007)

[44] H. Tanaka. J. Chem. Phys., 153 (13), 130901 (2020). DOI: $10.1063 / 5.0021045$

[45] L. Henry, M. Mezouar, G. Garbarino, D. Sifre, G. Weck, F. Datchi. Nature, 584, 382 (2020).

DOI: $10.1038 / \mathrm{s} 41586-020-2593-1$ 